\title{
A universal formula for generalized cardinal B-splines
}

\author{
Arash Amini ${ }^{\mathrm{a}}$, Ramtin Madani ${ }^{\mathrm{b}}$, Michael Unser ${ }^{\mathrm{b}}$ \\ ${ }^{a}$ EE department, Sharif University of Technology, Tehran, Iran. \\ ${ }^{b}$ Biomedical Imaging Group (BIG), École polytechnique fédérale de Lausanne (EPFL), \\ CH-1015 Lausanne, Switzerland.
}

\begin{abstract}
We introduce a universal and systematic way of defining a generalized Bspline based on a linear shift-invariant (LSI) operator L (a.k.a. Fourier multiplier). The generic form of the B-spline is $\beta_{\mathrm{L}}=\mathrm{L}_{\mathrm{d}} \mathrm{L}^{-1} \delta$ where $\mathrm{L}^{-1} \delta$ is the Green's function of $L$ and where $L_{d}$ is the discretized version of the operator that has the smallest-possible null space. The cornerstone of our approach is a main construction of $L_{d}$ in the form of an infinite product that is motivated by Weierstrass' factorization of entire functions. We show that the resulting Fourier-domain expression is compatible with the construction of all known B-splines. In the special case where $\mathrm{L}$ is the derivative operator (linked with piecewise-constant splines), our formula is equivalent to Euler's celebrated decomposition of $\operatorname{sinc}(x)=\frac{\sin (\pi x)}{\pi x}$ into an infinite product of polynomials. Our main challenge is to prove convergence and to establish continuity results for the proposed infinite-product representation. The ultimate outcome is the demonstration that the generalized $\mathrm{B}$-spline $\beta_{\mathrm{L}}$ generates a Riesz basis of the space of cardinal L-splines, where L is an essentially arbitrary pseudodifferential operator.
\end{abstract}

Keywords: Discrete Approximation, Differential Operator, Spline, Transfer Function.

\section{Introduction}

In mathematics and computer graphics, a spline is a function that is piecewise-polynomial of degree $n$ with $n-1$ continuous derivatives $[4,11]$. The points where the polynomial segments of the spline meet are called knots. The theory of splines was initiated by Schoenberg with the systematic investigation of the cardinal setting where the knots are positioned at 
the integers [10]. His fundamental result is that a cardinal polynomial spline admits a unique and stable expansion in a B-spline basis where the basis functions are shifted versions of one another. These B-splines are fundamental in the sense that they are the shortest-possible spline constituents. This minimal-support property makes them immensely useful in applications and numerical computations [3].

A powerful way of generalizing the notion of spline is by associating a particular brand of spline to some (pseudo) differential operator $\mathrm{L}[11,7]$ that, in this work, is assumed to be linear shift-invariant (LSI). Specifically, a function $f(x)$ is called a cardinal L-spline if

$$
\mathrm{L} f(x)=\sum_{k \in \mathbb{Z}} a[k] \delta(x-k)
$$

where $\delta$ is the Dirac impulse and $(a[k])_{k \in \mathbb{Z}}$ is a real-valued sequence of slow growth. The essence of this definition is that the application of the differential operator L uncovers the spline discontinuities: Their location is indicated by the Dirac impulses, which are positioned at the knots, while the size of the discontinuity at $x_{k}=k$ is encoded in $a[k]$.

The natural question that arises is whether or not Schoenberg's result on the existence of a B-spline basis extends for the generalized brands of L-splines. In other words, we want to investigate the possibility of specifying a generalized B-spline $\beta_{\mathrm{L}}(x)$ that is maximally localized - if possible, of minimum support - and that yields a stable expansion of any cardinal L-spline $f(x)$ such that

$$
f(x)=\sum_{k \in \mathbb{Z}} c[k] \beta_{\mathrm{L}}(x-k) .
$$

So far, the answer has been provided on a case-by-case basis together with rules for constructing B-splines for specific brands of splines. The most prominent examples are:

- Schoenberg's polynomial splines of order $m$ (or degree $(m-1))$ with $\mathrm{L}=\mathrm{D}^{m}$ where $\mathrm{D}=\frac{\mathrm{d}}{\mathrm{d} x}$ is the derivative operator $[10]$

- The exponential splines with $\mathrm{L}=\lambda_{m} \mathrm{D}^{m}+\cdots+\lambda_{1} \mathrm{D}+\mathrm{I}$ (exponential B-splines) $[2,8,13,1]$

- The fractional splines with $\mathrm{L}=\mathrm{D}^{\gamma}$ where $\mathrm{D}^{\gamma}$ is the fractional derivative of order $\gamma \in \mathbb{R}^{+}$with Fourier symbol $(\mathrm{i} \omega)^{\gamma}[12]$ 
A generic way of expressing $\beta_{\mathrm{L}}$ is

$$
\beta_{\mathrm{L}}=\mathrm{L}_{\mathrm{d}} \mathrm{L}^{-1} \delta=\sum_{k \in \mathbb{Z}} d[k] \rho_{\mathrm{L}}(\cdot-k)
$$

where $\mathrm{L}^{-1} \delta=\rho_{\mathrm{L}}$ is the Green's function of $\mathrm{L}$ and $\mathrm{L}_{\mathrm{d}}$ is a discrete approximation of the operator L (e.g., a finite-difference operator). While (1) gives some insight on the B-spline construction mechanism, it does not resolve the fundamental issue of the determination of an "optimum" set of weights $(d[k])$ for a given operator L; the latter has remained some kind of an art until now, requiring a special treatment for each particular brand of splines. Note that an equivalent form of (1) is

$$
\mathrm{L}_{\mathrm{d}} f(x)=\sum_{k \in \mathbb{Z}} d[k] f(x-k)=\left(\beta_{\mathrm{L}} * \mathrm{~L} f\right)(x)
$$

for any continuous and locally integrable function $f$, which points to the fact that the construction of B-splines is actually equivalent to the design of a "good" discrete approximation $\mathrm{L}_{\mathrm{d}}$ of the operator $\mathrm{L}$. Indeed, since $\mathrm{L}_{\mathrm{d}} f$ is a convolved version of $\mathrm{L} f$ with a B-spline, the best discretization is obtained when the convolution kernel is the most localized and closest (in an appropriate sense) to a Dirac impulse.

The purpose of this paper is to propose a principled approach to this general design problem. The outcome is a general formula for cardinal B-splines that works for a remarkably broad class of (pseudo-)differential operators. These are characterized by a Fourier-domain multiplier $\widehat{L}(\omega)$ that satisfies the admissibility conditions in Definition 2. The proposed framework is consistent with all known constructions and lends itself to the derivation of a number of fundamental properties; it also suggests new possibilities and extensions of spline theory. The only limiting assumption of the present Fourier-based formulation is that it is restricted to the cardinal setting where the data points are uniformly sampled on the integer grid.

\subsection{Notations}

In this paper, we reserve the notation i for the imaginary unit, and by $\operatorname{Re}(z)$ and $\operatorname{Im}(z)$ we represent the real and imaginary parts of a complex number $z$. The parameters $s \in \mathbb{C}$ and $\omega \in \mathbb{R}$ are used exclusively to index the Laplace and Fourier domains, respectively. The transfer function-or 
Laplace transform - of an LSI operator L is defined as

$$
L(s)=\int_{\mathbb{R}}(\mathrm{L} \delta)(x) \mathrm{e}^{s x} \mathrm{~d} x,
$$

where $\delta$ stands for the Dirac impulse. The set of all $s \in \mathbb{C}$ for which the above integral is absolutely convergent is called the region of convergence of $\mathrm{L}$, or simply $R O C_{\mathrm{L}}$. The frequency response of the operator is denoted by $\widehat{L}(\omega)=$ $\left.L(s)\right|_{s=\mathrm{i} \omega}$, if $R O C_{\mathrm{L}}$ includes the imaginary axis. Similarly, we represent the Fourier transform of a function $\beta$ (e.g., a B-spline) by $\widehat{\beta}$. Finally, we define $\mathcal{B}_{a}(z)$ to be the Euclidean open ball of radius $a \in \mathbb{R}^{+}$in the complex plane centered at $z \in \mathbb{C}$.

\subsection{Motivation}

The finite-difference operator is the most natural - and shortest - discrete approximation of the derivative operator. However, for a general operator $\mathrm{L}$, there is no consensus on the proper choice of $\mathrm{L}_{\mathrm{d}}$. A basic requirement for localization is that the null space of $\mathrm{L}_{\mathrm{d}}$ should include that of $\mathrm{L}$, but this constraint is not restrictive enough to uniquely determine $\mathrm{L}_{\mathrm{d}}$. Since the null space of L corresponds to the zeros of its transfer function $L(s)$, intuitively, one expects that $L_{\mathrm{d}}(s)=L(s) Q(s)$ (i.e., $L_{\mathrm{d}}(s)$ is divisible by $L(s)$ ), where $Q$ is a well-defined and bounded transfer function over $R O C_{\mathrm{L}}$. Due to the discrete nature of $\mathrm{L}_{\mathrm{d}}$, its transfer function is $\mathrm{i} 2 \pi$-periodic. Therefore, the zeros of $L(s+\mathrm{i} 2 \pi k)$ for any $k \in \mathbb{Z}$ should also be zeros of $L_{\mathrm{d}}(s)$. If the spacing between any two zeros of $L(s)$ is not an integer multiple of i $2 \pi$, then these zeros are all distinct. This observation suggests that $L_{\mathrm{d}}(s)$ should be divisible by $\prod_{k \in \mathbb{Z}} L(s+\mathrm{i} 2 \pi k)$ and, further, that the discrete approximation with the smallest null space is given by $\prod_{k \in \mathbb{Z}} L(s+i 2 \pi k)$. However, there are serious issues with this line of argument as this infinite product rarely converges. In Theorem 2.4, we refine this construction to obtain a valid $\mathrm{L}_{\mathrm{d}}$ and, in Theorem 2.7, we extend our result to specify the B-spline. For instance, if the frequency response $\widehat{L}$ is smooth (twice differentiable) and $\lim _{|\omega| \rightarrow \infty} \frac{\frac{d}{d \omega} \widehat{L}(\omega)}{\widehat{L}(\omega)}=0$, then the generalized B-spline is obtained as

$$
\widehat{\beta}_{\mathrm{L}}(\omega)=\prod_{k=1}^{\infty} \frac{\widehat{L}(\omega+2 \pi k) \widehat{L}(\omega-2 \pi k)}{G_{k}}
$$


if $\widehat{L}(\omega)$ is an even function, or as

$$
\widehat{\beta}_{\mathrm{L}}(\omega)=\mathrm{e}^{\mathrm{i} i} \frac{\omega}{2} \prod_{k=1}^{\infty} \frac{\widehat{L}(\omega+2 \pi k) \widehat{L}(\omega-2 \pi k)}{G_{k}}
$$

if $\widehat{L}(\omega)$ is an odd function, where

$$
G_{k}= \begin{cases}\widehat{L}(2 \pi k) \widehat{L}(-2 \pi k) & \text { if } \widehat{L}( \pm 2 \pi k) \neq 0 \\ 1 & \text { otherwise }\end{cases}
$$

Although this result applies to even- or odd-symmetric $\widehat{L}$, our construction in Theorem 2.7 is general and covers the asymmetric cases as well.

\section{Universal B-spline formula}

\subsection{Admissibility Conditions}

We now lay out the technical requirements on $\mathrm{L}$ that ensure the validity of our characterization.

Definition 2.1. Let $L$ be a linear operator with transfer function $L(s)$. We call L "discretizable" if there exist $x_{1}<x_{2}$ (possibly $\pm \infty$ ) and $0 \leq \Omega \in \mathbb{R}$ such that

(i) the $\mathrm{ROC}$ of $L(s)$ is of the form $\left\{s: x_{1}<\operatorname{Re}(s)<x_{2}\right\}$,

(ii) $L(s)$ is bounded on all compact subsets of its ROC,

(iii) $L(s)$ is holomorphic and nonzero at

$$
\left\{s: x_{1}<\operatorname{Re}(s)<x_{2},|\operatorname{Im}(s)|>\Omega\right\},
$$

(iv) there exist constants $0<c, \epsilon \in \mathbb{R}$ such that

$$
\forall s \in R O C_{\mathrm{L}},|\operatorname{Im}(s)|>\Omega:\left|\frac{\mathrm{d}^{2}}{\mathrm{~d} s^{2}} \log L(s)\right| \leq \frac{c}{|\operatorname{Im}(s)|^{1+\epsilon}} .
$$

Remark 2.2. The use of log for complex numbers inherently induces an ambiguity within an additive integer multiple of i2 $\pi$ and there are numerous conventions (such as taking the principal branch) to overcome this ambiguity. In all cases, however, we shall have $\frac{\mathrm{d}}{\mathrm{d} s} \log L(s)=\frac{\frac{\mathrm{d}}{\mathrm{d} s} L(s)}{L(s)}$. Therefore, Condition (iv) of Definition 2.1 is not influenced by the specific definition of the complex log. 
We note that the requirements on $L(s)$ in Definition 2.1 are not very restrictive: For instance, they are satisfied by all polynomials, rational transfer functions, power functions, as well as exponentials.

Since our specification of B-splines is done in the Fourier domain, we also need to state the requirements for admissibility in terms of the frequency response $\widehat{L}$. Additionally, we want to ensure that $\beta_{\mathrm{L}}$ generates a Riesz basis, which leads to the assumptions listed in Definition 2.3.

Definition 2.3. Let the continuous function $\widehat{L}(\omega)=L(\mathrm{i} \omega)$ be the Fourier multiplier associated with the operator L. We call L "spline-admissible" if there exists $0 \leq \Omega \in \mathbb{R}$ such that

(i) $\widehat{L}(\omega)$ has a finite number of (distinct) zeros, all included in $[-\Omega, \Omega]$, and the spacing between no two of them is an integer multiple of $2 \pi$;

(ii) $\widehat{L}(\omega)$ is twice differentiable at all $|\omega|>\Omega$ and there exist constants $0<c_{1}, \epsilon_{1} \in \mathbb{R}$ such that

$$
\forall \omega,|\omega|>\Omega: \quad\left|\frac{\mathrm{d}^{2}}{\mathrm{~d} \omega^{2}} \log \widehat{L}(\omega)\right| \leq \frac{c_{1}}{|\omega|^{1+\epsilon_{1}}} ;
$$

(iii) there exist constants $0<c_{2}, \epsilon_{2} \in \mathbb{R}$ such that

$$
\forall \omega,|\omega|>\Omega: \quad|\widehat{L}(\omega)| \geq c_{2}|\omega|^{\frac{1}{2}+\epsilon_{2}} .
$$

We observe that "spline-admissibility" is a stronger requirement than "discretizability" under the assumption that L has a well-defined frequency response. For instance, Definition 2.3 imposes the extra constraint (iii) on the minimum asymptotic growth rate of $\widehat{L}(\omega)$. As for the smoothness requirement of the two definitions, we observe that $L(s)$ is a complex function for which first-order differentiability implies differentiability of any order.

\subsection{Fourier-domain characterization of generalized B-spline}

Theorem 2.4. Let $\mathrm{L}$ be discretizable according to Definition 2.1. Then, it is possible to set $\sigma, g_{k}, \theta_{k} \in \mathbb{C}$ with $k \in \mathbb{Z}$ such that

(i) the infinite product

$$
\mathrm{e}^{\frac{\mathrm{i} \sigma}{2 \pi} s^{2}} \prod_{k \in \mathbb{Z}} \frac{L(s+\mathrm{i} 2 \pi k)}{g_{k} \mathrm{e}^{s \theta_{k}}}=\mathrm{e}^{\frac{\mathrm{i} \sigma}{2 \pi} s^{2}} \lim _{n \rightarrow+\infty} \prod_{k=-n}^{n} \frac{L(s+\mathrm{i} 2 \pi k)}{g_{k} \mathrm{e}^{s \theta_{k}}}
$$


converges uniformly on all compact subsets of $R O C_{\mathrm{L}}$ to an $\mathrm{i} 2 \pi$-periodic transfer function $L_{\mathrm{d}}(s)$;

(ii) $L_{\mathrm{d}}(s)$ is non-trivial in the sense that it is non-zero whenever $0 \notin\{L(s+$ $\mathrm{i} 2 \pi k)\}_{k} ;$ and

(iii) given there exists an open ball $\mathcal{B}_{\epsilon}\left(s_{0}\right) \subset R O C_{\mathrm{L}}$ such that $\bigcup_{k \in \mathbb{Z}}\left\{\mathcal{B}_{\epsilon}\left(s_{0}\right)+\right.$ $\mathrm{i} 2 \pi k\}$ contains no zeros of $L(s)$, any two discretizations $\mathrm{L}_{\mathrm{d}}$ and $\widetilde{\mathrm{L}}_{\mathrm{d}}$ of $\mathrm{L}$ that fulfill (i) and (ii) (possibly with different choices of $g_{k}, \theta_{k}$ ) shall satisfy

$$
\frac{\widetilde{L}_{\mathrm{d}}(s)}{L_{\mathrm{d}}(s)}=\alpha \mathrm{e}^{s m},
$$

for some $\alpha \in \mathbb{C} \backslash\{0\}$ and $m \in \mathbb{Z}$.

Theorem 2.4 shows that the infinite product alluded to in Section 1.2 is well-defined provided that the terms are properly scaled.

Remark 2.5. The choice of $\sigma$ in (2) that ensures a nontrivial and i $2 \pi$-periodic transfer function $L_{\mathrm{d}}(s)$ is unique. However, there are infinitely many settings for $g_{k}, \theta_{k}$ that result in different $L_{\mathrm{d}}(s)$. Part (iii) of Theorem 2.4 shows that the resulting transfer functions are tightly related to each other.

Remark 2.6. The Gaussian constant $\sigma$ vanishes when

$$
\lim _{|\omega| \rightarrow \infty} \frac{\frac{\mathrm{d}}{\mathrm{d} \omega} \widehat{L}(\omega)}{\widehat{L}(\omega)}=0 .
$$

This condition is not too restrictive as it applies to all $\widehat{L}$ frequency multipliers with polynomial growth. In particular, this covers the conventional range of spline theory.

Theorem 2.7. Let $\mathrm{L}$ be spline-admissible as specified in Definition 2.3. According to Theorem 2.4, it is possible to set $\sigma, g_{k}, \theta_{k} \in \mathbb{C}$ and define $\mathrm{L}_{\mathrm{d}}$ by

$$
\widehat{L}_{\mathrm{d}}(\omega)=\mathrm{e}^{-\frac{\mathrm{i} \sigma}{2 \pi} \omega^{2}} \prod_{k \in \mathbb{Z}} \frac{\widehat{L}(\omega+2 \pi k)}{g_{k} \mathrm{e}^{\mathrm{i} \omega \theta_{k}}} .
$$

Then, the generalized B-spline

$$
\widehat{\beta}_{\mathrm{L}}(\omega)=\frac{\widehat{L}_{\mathrm{d}}(\omega)}{\widehat{L}(\omega)}
$$


fulfills the Riesz-basis condition in the sense that there exist $0<m \leq M<\infty$ such that

$$
m\left\|\alpha_{n}\right\|_{\ell_{2}}^{2} \leq\left\|\sum_{n} \alpha_{n} \beta_{\mathrm{L}}(x-n)\right\|_{L_{2}}^{2} \leq M\left\|\alpha_{n}\right\|_{\ell_{2}}^{2}
$$

for any sequence $\left\{\alpha_{n}\right\} \in \ell_{2}$.

\subsection{Illustrative Example}

To illustrate the significance of the results, let us study the simple example $\mathrm{L}=\mathrm{D}+\lambda \mathrm{I}$, where $\lambda \in \mathbb{C}$ is a constant. The ROC of $L(s)=s+\lambda$ is the whole complex plane and the transfer function is holomorphic everywhere. Furthermore, whenever $|\operatorname{Im}(s)|>|\operatorname{Im}(\lambda)|$, we have that

$$
\left\{\begin{array}{l}
\frac{\mathrm{d}}{\mathrm{d} s} \log L(s)=\frac{1}{s+\lambda} \\
\frac{\mathrm{d}^{2}}{\mathrm{~d} s^{2}} \log L(s)=\frac{-1}{(s+\lambda)^{2}} .
\end{array}\right.
$$

It is not difficult to verify that $L(s)$ is discretizable according to Definition 2.1 with $x_{1}=-\infty, x_{2}=+\infty, \Omega=1+|\operatorname{Im}(\lambda)|, \epsilon=1$, and $c=(1+|\operatorname{Im}(\lambda)|)^{2}$. To find the factorization of $L_{\mathrm{d}}$ as in (2), one can apply the constructive proof of Theorem 2.4 which provides a systematic way of finding $\sigma, g_{k}, \theta_{k}$, except for $\theta_{0}$. Alternatively, Theorem 2.4 implies that any set of $\sigma, g_{k}, \theta_{k}$ guaranteeing convergence and periodicity of (2) results in almost the same operator $L_{d}$. Here, we introduce

$$
\begin{aligned}
& \sigma=0, \\
& g_{k}= \begin{cases}\mathrm{i} 2 \pi k \exp \left(\frac{-\mathrm{i} \lambda}{2 \pi k}\right) & k \neq 0, \\
\exp (\lambda / 2) & k=0,\end{cases} \\
& \theta_{k}= \begin{cases}\frac{-\mathrm{i}}{2 \pi k} & k \neq 0, \\
\frac{1}{2} & k=0 .\end{cases}
\end{aligned}
$$

Thus, the Gaussian term $\exp \left(\frac{\mathrm{i} \sigma}{2 \pi} s^{2}\right)$ in (2) simplifies to 1 and the factors in the infinite product, except for $k=0$, take the form

$$
\frac{L(s+\mathrm{i} 2 \pi k)}{g_{k} \mathrm{e}^{s \theta_{k}}}=\frac{s+\lambda+\mathrm{i} 2 \pi k}{\mathrm{i} 2 \pi k \exp \left(\frac{-\mathrm{i}(s+\lambda)}{2 \pi k}\right)}=\left(1-\frac{\mathrm{i}(s+\lambda)}{2 \pi k}\right) \mathrm{e}^{\frac{\mathrm{i}(s+\lambda)}{2 \pi k}} .
$$


Based on the inequality $\left|(1-z) \mathrm{e}^{z}-1\right| \leq|z|^{2}$ for $|z| \leq 1$, we deduce that

$$
\left|\frac{L(s+\mathrm{i} 2 \pi k)}{g_{k} \mathrm{e}^{s \theta_{k}}}-1\right|=\mathcal{O}\left(\frac{1}{k^{2}}\right) \text {. }
$$

This reveals that $\frac{\mathrm{L}(s+\mathrm{i} 2 \pi k)}{g_{k} \mathrm{e}^{\theta_{k}}}-1$ is absolutely summable, which guarantees convergence of (2), in agreement with the statement in Theorem 2.4.

To examine the periodicity, we evaluate the infinite product

$$
\begin{aligned}
L_{\mathrm{d}}(s) & =\frac{L(s)}{g_{0} \mathrm{e}^{s \theta_{0}}} \prod_{k=1}^{\infty} \frac{L(s+\mathrm{i} 2 \pi k)}{g_{k} \mathrm{e}^{s \theta_{k}}} \frac{L(s-\mathrm{i} 2 \pi k)}{g_{-k} \mathrm{e}^{s \theta_{-k}}} \\
& =\frac{s+\lambda}{\mathrm{e}^{\frac{1}{2}(s+\lambda)}} \prod_{k=1}^{\infty}\left(1-\frac{\left(\frac{1}{2} \mathrm{i}(s+\lambda)\right)^{2}}{(\pi k)^{2}}\right) \\
& =-2 \mathrm{ie}^{-\frac{1}{2}(s+\lambda)} \sin \left(\frac{1}{2} \mathrm{i}(s+\lambda)\right) \\
& =\mathrm{e}^{-\frac{1}{2}(s+\lambda)}\left(\mathrm{e}^{\frac{1}{2}(s+\lambda)}-\mathrm{e}^{-\frac{1}{2}(s+\lambda)}\right) \\
& =1-\mathrm{e}^{-\lambda} \mathrm{e}^{-s}
\end{aligned}
$$

where we used Euler's celebrated formula

$$
\sin (x)=x \prod_{k=1}^{\infty}\left(1-\left(\frac{x}{\pi k}\right)^{2}\right) .
$$

It is trivial to check that the final expression in (7) is i2 $\pi$-periodic. Moreover, (7) suggests the operator

$$
\mathrm{L}_{\mathrm{d}} f(x)=f(x)-\mathrm{e}^{-\lambda} f(x-1)
$$

as the discrete counterpart of $\mathrm{L}=\mathrm{D}+\lambda \mathrm{I}$. Finally, the corresponding B-spline is given by

$$
\beta_{\mathrm{L}}(x)=\mathcal{F}^{-1}\left\{\frac{1-\mathrm{e}^{-\mathrm{i} \omega-\lambda}}{\mathrm{i} \omega+\lambda}\right\}(x)=\mathrm{e}^{-\lambda x}\left(\mathbb{1}_{+}(x)-\mathbb{1}_{+}(x-1)\right),
$$

where $\mathcal{F}^{-1}$ denotes the inverse Fourier transform and $\mathbb{1}_{+}(\cdot)$ stands for the Heaviside step function. We observe that the expression in (8) matches the standard definition of exponential B-splines [13]. 


\section{Properties \& Generalization}

We shall now apply the theorems to infer a few useful properties of Bsplines.

Property 3.1. If $\mathrm{L}_{\mathrm{d}}$ and $\widetilde{\mathrm{L}}_{\mathrm{d}}$ are two discretizations of $\mathrm{L}$ and result in the B-splines $\beta_{\mathrm{L}}$ and $\widetilde{\beta}_{\mathrm{L}}$, respectively, Statement (iii) of Theorem 2.4 implies that

$$
\widetilde{\beta}_{\mathrm{L}}(x)=\alpha \beta_{\mathrm{L}}(x-m),
$$

where $\alpha \in \mathbb{C}$ and $m \in \mathbb{Z}$ are constants.

Property 3.2. The discretization process of Theorem 2.4 is multiplicative. In other words, if $\mathrm{L}_{1}$ and $\mathrm{L}_{2}$ are operators that satisfy Definition 2.1 and correspond to discretized operators $\mathrm{L}_{\mathrm{d}, 1}$ and $\mathrm{L}_{\mathrm{d}, 2}$, respectively, then $\mathrm{L}_{1} \mathrm{~L}_{2}$ also satisfies Definition 2.1 and $\mathrm{L}_{\mathrm{d}, 1} \mathrm{~L}_{\mathrm{d}, 2}$ is one of its valid discretizations. Further, if these two operators define the splines $\beta_{\mathrm{L}_{1}}$ and $\beta_{\mathrm{L}_{2}}$, respectively, then the spline associated with $\mathrm{L}_{1} \mathrm{~L}_{2}$ is

$$
\beta_{\mathrm{L}_{1} \mathrm{~L}_{2}}(x)=\left(\beta_{\mathrm{L}_{1}} * \beta_{\mathrm{L}_{2}}\right)(x) .
$$

In particular, this shows that the study of $\mathrm{L}=\mathrm{D}+\lambda \mathrm{I}$ is sufficient for determining the exponential B-splines associated with all differential operators of the form $\lambda_{m} \mathrm{D}^{m}+\cdots+\lambda_{1} \mathrm{D}+\mathrm{I}$, due to the fundamental theorem of algebra.

Property 3.3. Let $z_{0}, u \in \mathbb{C}$ with $|u|=1$ and $\operatorname{Re}\left(z_{0}\right) \geq 1$, and let the Fourier multiplier $\widehat{L}(\omega)$ be spline-admissible (Definition 2.3) such that $\frac{\widehat{L}(\omega)}{|\widehat{L}(\omega)|} \neq u$ for all $\omega$. Then, the operator of order $z_{0} \in \mathbb{C}$ defined as $\widehat{L}_{z_{0}}(\omega)=\widehat{L}(\omega)^{z_{0}}$, where

$$
\forall c \in \mathbb{C}: c^{z_{0}}:= \begin{cases}\exp \left(z_{0} \log |c|+\mathrm{i} z_{0} \arg ^{*}(c)\right) & c \neq 0, \\ 0 & c=0,\end{cases}
$$

and $\arg ^{*}(c) \in\left[\measuredangle u, \measuredangle u+2 \pi\left[\right.\right.$, is spline-admissible as well. Moreover, let $\beta_{\mathrm{L}}$ and $\beta_{\mathrm{L}_{z_{0}}}$ represent the splines generated by $\mathrm{L}$ and $\mathrm{L}_{z_{0}}$, respectively. Then, we have that

$$
\left|\widehat{\beta}_{\mathrm{L}_{0}}(\omega)\right|=\left|\widehat{\beta}_{\mathrm{L}}(\omega)\right|^{\operatorname{Re}\left(z_{0}\right)}
$$




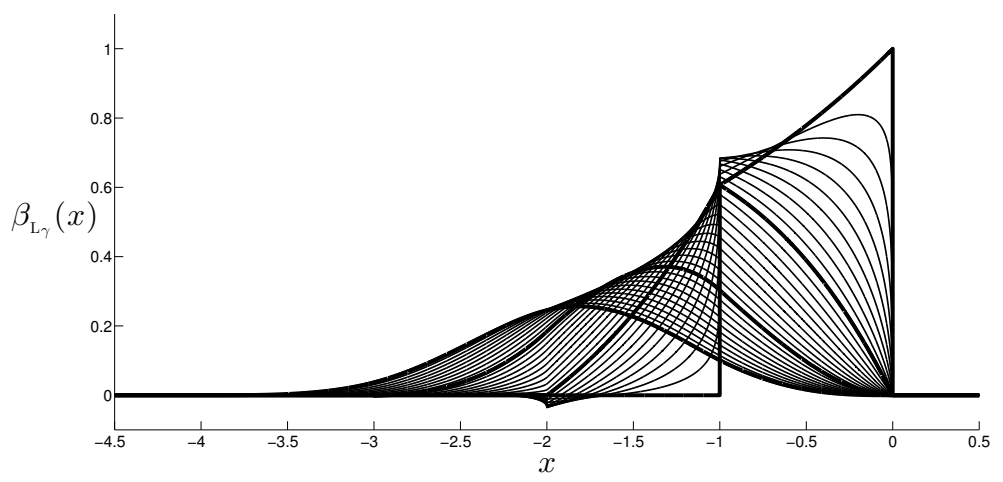

Figure 1: Fractional polynomial splines corresponding to $\widehat{L}(\omega)=(\mathrm{i} \omega-\lambda)^{\gamma}$ with $\lambda=0.5$ and $\gamma=1$ to 4 in steps of 0.1 (the support of the spline increases as $\gamma$ increases).

Example 3.4. The fractional-order splines of [12] are special cases of Property 3.3 when $\widehat{L}(\omega)=\mathrm{i} \omega$ and $z_{0}$ is a positive real no less than 1 . Similarly, the complex-order splines of [5] and [6] are obtained by setting $\widehat{L}(\omega)=\mathrm{i} \omega$ and $\widehat{L}(\omega)=\mathrm{i} \omega+\lambda$ with $\operatorname{Re}(\lambda) \geq 0$, respectively, for some complex order $\operatorname{Re}\left(z_{0}\right) \geq 1$. Note that $\frac{\mathrm{i} \omega+\lambda}{|\mathrm{i} \omega+\lambda|} \neq-1$ whenever $\operatorname{Re}(\lambda) \geq 0$.

Example 3.5. Let $\widehat{L}(\omega)=i \omega-\lambda$ (or $\mathrm{L}=\mathrm{D}-\lambda \mathrm{I})$ with $0<\operatorname{Re}(\lambda)$. It is easy to check that $\frac{\widehat{L}(\omega)}{|\widehat{L}(\omega)|} \neq 1$. Thus, as mentioned in Property 3.3, by modifying the definition of $\arg$ to $\arg ^{*}(c) \in\left[0,2 \pi\left[\right.\right.$, we can redefine $c^{z_{0}}$ to make $\widehat{L}(\omega)^{z_{0}}$ continuous. Thus,

$$
\begin{aligned}
\widehat{L}_{z_{0}}(\omega) & =(\mathrm{i} \omega-\lambda)^{z_{0}} \\
& =|\mathrm{i} \omega-\lambda|^{z_{0}} \exp \left(\mathrm{i} z_{0}\left(\pi-\tan ^{-1}\left(\frac{\omega-\operatorname{Im}(\lambda)}{\operatorname{Re}(\lambda)}\right)\right)\right) .
\end{aligned}
$$

To discretize $\widehat{L}_{z_{0}}$, similarly to the illustrative example, we introduce the following set of parameters:

$$
\begin{aligned}
\sigma & =0 \\
g_{k} & = \begin{cases}(\mathrm{i} 2 \pi k)^{z_{0}} \exp \left(\frac{\mathrm{i} z_{0} \lambda}{2 \pi k}\right) & k \neq 0, \\
\left.\exp \left(z_{0} \lambda / 2\right)\right) & k=0,\end{cases} \\
\theta_{k} & = \begin{cases}\frac{-\mathrm{i} z_{0}}{2 \pi k} & k \neq 0, \\
\frac{-z_{0}}{2} & k=0\end{cases}
\end{aligned}
$$


With this choice, the infinite product of (3) converges to

$$
\widehat{L}_{z_{0}, d}(\omega)=\left(\mathrm{e}^{\mathrm{i} \omega-\lambda}-1\right)^{z_{0}}
$$

as proven in the appendix. Next, because $\left|\mathrm{e}^{\mathrm{i} \omega-\lambda}\right|<1$, we can apply the binomial expansion in the form of

$$
\widehat{L}_{z_{0}, d}(\omega)=\sum_{k=0}^{\infty}\left(\begin{array}{c}
z_{0} \\
k
\end{array}\right)(-1)^{z_{0}-k} \mathrm{e}^{k(\mathrm{i} \omega-\lambda)},
$$

where $\left(\begin{array}{c}z_{0} \\ 0\end{array}\right)=1$ and $\left(\begin{array}{c}z_{0} \\ k\end{array}\right)=\frac{z_{0}\left(z_{0}-1\right) \cdots\left(z_{0}-k+1\right)}{k !}$. Thus, if $\rho_{\lambda}^{z_{0}}(x)$ denotes the inverse Fourier of $\left(\widehat{L}_{z_{0}}(\omega)\right)^{-1}$, we have that

$$
\begin{aligned}
\beta_{\mathrm{L}_{z_{0}}}(x) & =\mathcal{F}^{-1}\left\{\frac{\widehat{L}_{z_{0}, d}(\omega)}{\widehat{L}_{z_{0}}(\omega)}\right\}(x) \\
& =(-1)^{z_{0}} \sum_{k=0}^{\infty}\left(\begin{array}{c}
z_{0} \\
k
\end{array}\right)\left(-\mathrm{e}^{-\lambda}\right)^{k} \rho_{\lambda}^{z_{0}}(x+k) .
\end{aligned}
$$

For $z_{0}=\gamma \in \mathbb{R}_{\geq 1}$, we know that $\rho_{\lambda}^{\gamma}(x)=\frac{\mathrm{e}^{\lambda x} x_{-}^{\gamma-1}}{(-1)^{\gamma} \Gamma\left(z_{0}\right)}$, where $x_{-}^{\gamma-1}$ is 0 for $x>0$, and $(-x)^{\gamma-1}$ when $x \leq 0$, in which case (15) simplifies to

$$
\beta_{\mathrm{L}_{\gamma}}(x)=\frac{\mathrm{e}^{\lambda x}}{\Gamma(\gamma)} \sum_{k=0}^{\infty}(-1)^{k}\left(\begin{array}{l}
\gamma \\
k
\end{array}\right)(x+k)_{-}^{\gamma-1} .
$$

In Figure 1, we have plotted the spline functions for $\lambda=\frac{1}{2}$ and various values of $\gamma$.

Example 3.6. So far, all the studied Fourier multipliers had polynomial growth. Here, we investigate the exponentially increasing $\widehat{L}(\omega)=\cosh (\omega)$ which corresponds to $L(s)=\cos (s)$. Since $\widehat{L}(\omega) \in \mathbb{R}_{\geq 1}$, there is no difficulty in defining $\widehat{L}_{z_{0}}(\omega)=\cosh (\omega)^{z_{0}}$. One can also check that $\widehat{L}_{z_{0}}(\omega)$ is discretizable for $\operatorname{Re}\left(z_{0}\right)>0$ with the following setting:

$$
\sigma=-\mathrm{i} z_{0}, \quad g_{k}=\cosh (2 \pi k)^{z_{0}}, \quad \theta_{k}=-\mathrm{i} z_{0} \tanh (2 \pi k) .
$$

We have plotted in Figure 2 the novel B-splines arising from $\widehat{L}_{\gamma}(\omega)$ for various values of $\gamma \in \mathbb{R}^{+}$. Since the inverse Fourier of $\left(\widehat{L}_{\gamma}(\omega)\right)^{-1}$ is given by $\rho^{\gamma}(x)=$ $\frac{2^{\gamma}}{4 \pi \Gamma(\gamma)}\left|\Gamma\left(\frac{\gamma+\mathrm{i} x}{2}\right)\right|^{2}$, the corresponding B-spline is a linear combination of integer shifts of $\rho^{\gamma}(x)$. 


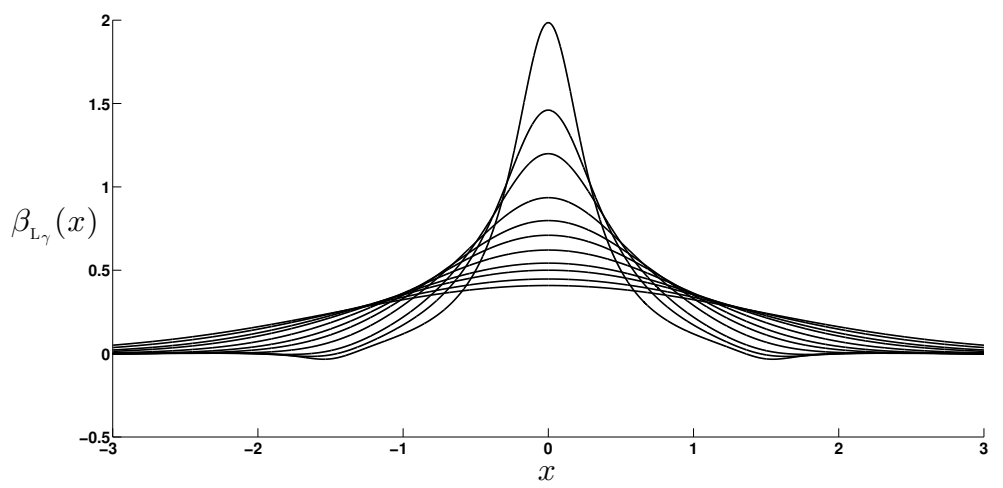

Figure 2: Fractional cosh splines corresponding to $\widehat{L}_{\gamma}(\omega)=\cosh (\omega)^{\gamma}$ where $\gamma \in$ $\{0.2,0.3,0.4,0.6,0.8,1,1.3,1.7,2,2.5,3\}$ (the peak of the B-spline falls as $\gamma$ increases).

\section{Proofs}

Let $\mathcal{Z}^{+}=R O C_{\mathrm{L}} \cap\{s, \operatorname{Im}(s)>\Omega\}$ and $\mathcal{Z}^{-}=R O C_{\mathrm{L}} \cap\{s, \operatorname{Im}(s)<$ $-\Omega\}$. Since both $\mathcal{Z}^{+}$and $\mathcal{Z}^{-}$are connected open subsets of the complex plane on which $L(s)$ is nonzero and holomorphic, one can define holomorphic branches of $\log L(s)$ on these regions [9]; these are denoted by $\mathrm{H}^{+}(s)$ and $H^{-}(s)$, respectively.

Definition 4.1. For any given $s_{0}, s \in R O C_{\mathrm{L}}$, we define

$$
\begin{aligned}
& k^{+}\left(s_{0}, s\right)=1+\frac{1}{2 \pi}\left(\Omega-\min \left(\operatorname{Im}\left(s_{0}\right), \operatorname{Im}(s)\right)\right), \\
& k^{-}\left(s_{0}, s\right)=-1-\frac{1}{2 \pi}\left(\Omega+\max \left(\operatorname{Im}\left(s_{0}\right), \operatorname{Im}(s)\right)\right) .
\end{aligned}
$$

Lemmas 4.2 and 4.3 lay down the foundations for the proofs given below.

Lemma 4.2. Let $s_{0} \in R O C_{\mathrm{L}}$ and $k \in \mathbb{Z}$. Then, as $k \rightarrow+\infty$, the terms $\left.\frac{\mathrm{d}}{\mathrm{d} s} H^{+}(s)\right|_{s=s_{0}+\mathrm{i} 2 \pi k}$ and $\left.\frac{\mathrm{d}}{\mathrm{d} s} H^{-}(s)\right|_{s=s_{0}-\mathrm{i} 2 \pi k}$ converge to finite values $\dot{H}_{+\infty}^{\left(s_{0}\right)}$ and $\dot{H}_{-\infty}^{\left(s_{0}\right)}$, respectively.

Lemma 4.3. Let $s_{0}, s \in R O C_{\mathrm{L}}$ and $k \in \mathbb{Z}$ such that either $k \geq k^{+}\left(s_{0}, s\right)$ or 
$k \leq k^{-}\left(s_{0}, s\right)$. Then, the function $q^{\left(s_{0}\right)}(s, k)$ defined by

$$
q^{\left(s_{0}\right)}(s, k)=\left\{\begin{aligned}
& H^{+}(s+\mathrm{i} 2 \pi k)-H^{+}\left(s_{0}+\mathrm{i} 2 \pi k\right) \\
&-\left.\left(s-s_{0}\right) \frac{\mathrm{d}}{\mathrm{d} s} H^{+}(s)\right|_{s=s_{0}+\mathrm{i} 2 \pi k}, k \geq k^{+}\left(s_{0}, s\right), \\
& H^{-}(s+\mathrm{i} 2 \pi k)-H^{-}\left(s_{0}+\mathrm{i} 2 \pi k\right) \\
&-\left.\left(s-s_{0}\right) \frac{\mathrm{d}}{\mathrm{d} s} H^{-}(s)\right|_{s=s_{0}+\mathrm{i} 2 \pi k}, \quad k \leq k^{-}\left(s_{0}, s\right),
\end{aligned}\right.
$$

is continuous with respect to $s$ as long as $k$ satisfies one of the inequalities, and

$$
\left|q^{\left(s_{0}\right)}(s, k)\right| \leq \frac{c\left|s-s_{0}\right|^{2}}{\min \left(\left|\operatorname{Im}\left(s_{0}\right)+2 \pi k\right|^{1+\epsilon},|\operatorname{Im}(s)+2 \pi k|^{1+\epsilon}\right)} .
$$

Moreover,

(i) $\lim _{|k| \rightarrow \infty} q^{\left(s_{0}\right)}(s, k)=0$, and

(ii) $\sum_{k \geq k^{+}\left(s_{0}, s\right)}\left|q^{\left(s_{0}\right)}(s, k)\right|+\sum_{k \leq k-\left(s_{0}, s\right)}\left|q^{\left(s_{0}\right)}(s, k)\right|$ converges to a finite value.

\subsection{Proof of Lemma 4.2}

Proof. We take advantage of Condition (iv) of Definition 2.1 that for all $k \geq k^{+}\left(s_{0}, 0\right)$, we have

$$
\left|\frac{\mathrm{d}^{2}}{\mathrm{~d} s^{2}} H^{+}(s)\right|_{s=s_{0}+\mathrm{i} 2 \pi k}=\left|\frac{\mathrm{d}^{2}}{\mathrm{~d} s^{2}} \log L(s)\right|_{s=s_{0}+\mathrm{i} 2 \pi k} \leq \frac{c}{\left|\operatorname{Im}\left(s_{0}\right)+2 \pi k\right|^{1+\epsilon}} .
$$

This allows us to write that

$$
\begin{aligned}
\mid\left(\left.\lim _{k \rightarrow \infty} \frac{\mathrm{d}}{\mathrm{d} s} H^{+}(s)\right|_{s=s_{0}+\mathrm{i} 2 \pi k}\right) & -\left.\frac{\mathrm{d}}{\mathrm{d} s} H^{+}(s)\right|_{s=s_{0}+\mathrm{i} 2 \pi k^{+}\left(s_{0}, 0\right)} \mid \\
& =\left|\int_{k^{+}\left(s_{0}, 0\right)}^{\infty} \frac{\mathrm{d}^{2}}{\mathrm{~d} s^{2}} H^{+}(s)\right|_{s=s_{0}+\mathrm{i} 2 \pi k} \mathrm{~d} k \mid \\
& \leq \int_{k^{+}\left(s_{0}, 0\right)}^{\infty} \frac{c}{\left|\operatorname{Im}\left(s_{0}\right)+2 \pi k\right|^{1+\epsilon}} \mathrm{d} k<\infty .
\end{aligned}
$$

Thus, $\left.\lim _{k \rightarrow \infty} \frac{\mathrm{d}}{\mathrm{d} s} H^{+}(s)\right|_{s=s_{0}+\mathrm{i} 2 \pi k}$ exists and is finite. Similarly, for all $k \geq$ $-k^{-}\left(s_{0}, 0\right)$, Condition (iv) of Definition 2.1 results in

$$
\left|\frac{\mathrm{d}^{2}}{\mathrm{~d} s^{2}} H^{-}(s)\right|_{s=s_{0}-\mathrm{i} 2 \pi k}=\left|\frac{\mathrm{d}^{2}}{\mathrm{~d} s^{2}} \log L(s)\right|_{s=s_{0}-\mathrm{i} 2 \pi k} \leq \frac{c}{\left|2 \pi k-\operatorname{Im}\left(s_{0}\right)\right|^{1+\epsilon}},
$$

which again shows that $\left.\lim _{k \rightarrow \infty} \frac{\mathrm{d}}{\mathrm{d} s} H^{-}(s)\right|_{s=s_{0}-\mathrm{i} 2 \pi k}$ exists and is finite. 


\subsection{Proof of Lemma 4.3}

Proof. For $k \geq k^{+}\left(s_{0}, s\right)$, both $s_{0}+\mathrm{i} 2 \pi k$ and $s+\mathrm{i} 2 \pi k$ are located inside $\mathcal{Z}^{+}$. Thus, the function $\mathrm{H}^{+}$is well-defined on the line connecting these two points (similar arguments apply for $k \leq k^{-}\left(s_{0}, s\right)$ ). The continuity of $q^{\left(s_{0}\right)}(s, k)$ follows from the smoothness (holomorphicity) of $\mathrm{H}^{+}$and $\mathrm{H}^{-}$. To avoid the complication of working with the complex-valued function $\mathrm{H}^{+}$(similarly, $\mathrm{H}^{-}$), we focus on the two real-valued functions $R^{+}, I^{+}:[0,1] \rightarrow \mathbb{R}$ defined as

$$
\begin{aligned}
R^{+}(t) & =\operatorname{Re}\left(H^{+}\left(\left(s-s_{0}\right) t+\left(s_{0}+\mathrm{i} 2 \pi k\right)\right)\right), \\
I^{+}(t) & =\operatorname{Im}\left(H^{+}\left(\left(s-s_{0}\right) t+\left(s_{0}+\mathrm{i} 2 \pi k\right)\right)\right) .
\end{aligned}
$$

In fact, by varying $t$ from 0 to 1 , the point $\left(s-s_{0}\right) t+\left(s_{0}+\mathrm{i} 2 \pi k\right)$ moves away from $s_{0}+\mathrm{i} 2 \pi k$ towards $s+\mathrm{i} 2 \pi k$ on the line that connects the two points. As $\mathrm{H}^{+}$is holomorphic on this line, the real-valued functions $R^{+}$and $I^{+}$are continuous and differentiable of any order (particularly, second order). By applying the Lagrange form of Taylor's theorem, we obtain that

$$
\left\{\begin{array}{l}
R^{+}(1)=R^{+}(0)+\left.\frac{\mathrm{d}}{\mathrm{d} t} R^{+}(t)\right|_{t=0}+\left.\frac{1}{2} \frac{\mathrm{d}^{2}}{\mathrm{~d} t^{2}} R^{+}(t)\right|_{t=\xi}, \\
I^{+}(1)=I^{+}(0)+\left.\frac{\mathrm{d}}{\mathrm{d} t} I^{+}(t)\right|_{t=0}+\left.\frac{1}{2} \frac{\mathrm{d}^{2}}{\mathrm{~d} t^{2}} I^{+}(t)\right|_{t=\zeta}
\end{array}\right.
$$

where $\xi, \zeta$ are real numbers in $[0,1]$. The latter result can be rewritten in terms of $H^{+}$as

$$
\begin{aligned}
H^{+}(s+\mathrm{i} 2 \pi k)= & H^{+}\left(s_{0}+\mathrm{i} 2 \pi k\right)+\left.\left(s-s_{0}\right) \frac{\mathrm{d}}{\mathrm{d} s} H^{+}(s)\right|_{s=s_{0}+\mathrm{i} 2 \pi k} \\
& +\operatorname{Re}\left(\left.\frac{\left(s-s_{0}\right)^{2}}{2} \frac{\mathrm{d}^{2}}{\mathrm{~d} s^{2}} H^{+}(s)\right|_{s=\left(s-s_{0}\right) \xi+s_{0}+\mathrm{i} 2 \pi k}\right) \\
& +\mathrm{i} \operatorname{Im}\left(\left.\frac{\left(s-s_{0}\right)^{2}}{2} \frac{\mathrm{d}^{2}}{\mathrm{~d} s^{2}} H^{+}(s)\right|_{s=\left(s-s_{0}\right) \zeta+s_{0}+\mathrm{i} 2 \pi k}\right)
\end{aligned}
$$

or, equivalently, as

$$
\begin{aligned}
q^{\left(s_{0}\right)}(s, k)= & \operatorname{Re}\left(\left.\frac{\left(s-s_{0}\right)^{2}}{2} \frac{\mathrm{d}^{2}}{\mathrm{~d} s^{2}} H^{+}(s)\right|_{s=\left(s-s_{0}\right) \xi+s_{0}+\mathrm{i} 2 \pi k}\right) \\
& +\mathrm{i} \operatorname{Im}\left(\left.\frac{\left(s-s_{0}\right)^{2}}{2} \frac{\mathrm{d}^{2}}{\mathrm{~d} s^{2}} H^{+}(s)\right|_{s=\left(s-s_{0}\right) \zeta+s_{0}+\mathrm{i} 2 \pi k}\right) .
\end{aligned}
$$


This implies that

$$
\begin{aligned}
& \left|q^{\left(s_{0}\right)}(s, k)\right| \leq \frac{\left|s-s_{0}\right|^{2}}{2}\left(\left|\frac{\mathrm{d}^{2}}{\mathrm{~d} s^{2}} H^{+}(s)\right|_{s=\left(s-s_{0}\right) \xi+s_{0}+\mathrm{i} 2 \pi k}+\left|\frac{\mathrm{d}^{2}}{\mathrm{~d} s^{2}} H^{+}(s)\right|_{s=\left(s-s_{0}\right) \zeta+s_{0}+\mathrm{i} 2 \pi k}\right) \\
& \leq \frac{\left|s-s_{0}\right|^{2}}{2}\left(\frac{c}{\left|\xi \operatorname{Im}(s)+(1-\xi) \operatorname{Im}\left(s_{0}\right)+2 \pi k\right|^{1+\epsilon}}\right. \\
& \left.+\frac{c}{\left|\zeta \operatorname{Im}(s)+(1-\zeta) \operatorname{Im}\left(s_{0}\right)+2 \pi k\right|^{1+\epsilon}}\right) \\
& \leq \frac{c\left|s-s_{0}\right|^{2}}{\min \left(\left|\operatorname{Im}\left(s_{0}\right)+2 \pi k\right|^{1+\epsilon},|\operatorname{Im}(s)+2 \pi k|^{1+\epsilon}\right)} \text {, }
\end{aligned}
$$

where we used Condition (iv) of Definition 2.1 for $H^{+}=\log L$. The argument for $k \leq k^{-}\left(s_{0}, s\right)$ is similar and can be achieved by replacing $H^{+}$with $H^{-}$in the proof. The statements (i) and (ii) are direct consequences of inequality (20).

\subsection{Proof of Theorem 2.4.}

Proof. Here, we adopt a constructive approach. By assuming that Conditions (ii)-(iv) of Definition 2.1 are met, we introduce a set of constants $g_{k}, \theta_{k}$, with the exception of $\theta_{0}$, that guarantees uniform convergence of the infinite product. Then, we demonstrate how the periodicity of $\mathrm{L}_{\mathrm{d}}$ can be established by properly setting $\sigma$ and $\theta_{0}$. Note that the choice of $\sigma, \theta_{0}^{\left(s_{0}\right)}$ does not impact the status of convergence, as long as the chosen quantities are finite.

For clarity, we have divided the proof into four parts.

\section{Parameter Setting}

Let us fix $s_{0} \in R O C_{\mathrm{L}}$. For instance, if $0 \in R O C_{\mathrm{L}}$, then it is simplest to set $s_{0}=0$. Further, let $k_{0}$ be an integer no less than $\left|k^{+}\left(s_{0}, s_{0}\right)\right|$ and $\left|k^{-}\left(s_{0}, s_{0}\right)\right|$. For $k \in \mathbb{Z}$ define

$$
g_{k}^{\left(s_{0}\right)}= \begin{cases}L\left(s_{0}+\mathrm{i} 2 \pi k\right) \exp \left(-\left.s_{0} \frac{\mathrm{d}}{\mathrm{d} s} H^{+}(s)\right|_{s=s_{0}+\mathrm{i} 2 \pi k}\right) & k \geq k_{0} \\ 1 & |k|<k_{0} \\ L\left(s_{0}+\mathrm{i} 2 \pi k\right) \exp \left(-\left.s_{0} \frac{\mathrm{d}}{\mathrm{d} s} H^{-}(s)\right|_{s=s_{0}+\mathrm{i} 2 \pi k}\right) & k \leq-k_{0}\end{cases}
$$

and

$$
\theta_{k}^{\left(s_{0}\right)}= \begin{cases}\left.\frac{\mathrm{d}}{\mathrm{d} s} H^{+}(s)\right|_{s=s_{0}+\mathrm{i} 2 \pi k}=\frac{\left.\frac{\mathrm{d}}{\mathrm{d} s} L(s)\right|_{s=s_{0}+\mathrm{i} 2 \pi k}}{L\left(s_{0}+\mathrm{i} 2 \pi k\right)} & k \geq k_{0}, \\ 0 & 0<|k|<k_{0} \\ \left.\frac{\mathrm{d}}{\mathrm{d} s} H^{-}(s)\right|_{s=s_{0}+\mathrm{i} 2 \pi k}=\frac{\left.\frac{\mathrm{d}}{\mathrm{d} s} L(s)\right|_{s=s_{0}+\mathrm{i} 2 \pi k}}{L\left(s_{0}+\mathrm{i} 2 \pi k\right)} & k \leq-k_{0} .\end{cases}
$$


Further, based on Lemma 4.2, we set

$$
\sigma=\frac{\dot{H}_{+\infty}^{\left(s_{0}\right)}-\dot{H}_{-\infty}^{\left(s_{0}\right)}}{2}
$$

In particular, if $\frac{\frac{\mathrm{d}}{\mathrm{d} \omega} \widehat{L}(\omega)}{\widehat{L}(\omega)} \stackrel{|\omega| \rightarrow \infty}{\longrightarrow} 0$, then $\dot{H}_{+\infty}^{(0)}=\dot{H}_{-\infty}^{(0)}=0$, which yields $\sigma=0$ (Remark 2.6).

\section{Proof of Uniform Convergence}

We shall now prove that the corresponding infinite product in (2) converges for all permitted choices of $s_{0}$. Let $\mathcal{T}$ be a compact subset of $R O C_{\mathrm{L}}$. Therefore,

$$
\begin{aligned}
k_{\mathcal{T}}^{+} & =\sup \left\{k^{+}\left(s_{0}, s\right) \mid s \in \mathcal{T}\right\}, \\
k_{\mathcal{T}}^{-} & =\inf \left\{k^{-}\left(s_{0}, s\right) \mid s \in \mathcal{T}\right\}, \text { and } \\
d_{\mathcal{T}}^{\left(s_{0}\right)} & =\sup \left\{\left|s-s_{0}\right|^{2} \mid s \in \mathcal{T}\right\}
\end{aligned}
$$

are all finite. To unify $k_{\mathcal{T}}^{+}$and $k_{\mathcal{T}}^{-}$in our arguments, we further define $k_{\mathcal{T}}^{ \pm}=\max \left(\left|k_{\mathcal{T}}^{+}\right|,\left|k_{\mathcal{T}}^{-}\right|\right)$. Thus, for all $s \in \mathcal{T}$ and $k \in \mathbb{Z}$ such that $|k| \geq k_{\mathcal{T}}^{ \pm}$, the pair $(s, k)$ is a valid input argument for the function $q^{\left(s_{0}\right)}$ of Lemma 4.3. This yields

$\forall s \in \mathcal{T}: \prod_{\substack{k \in \mathbb{Z} \\ k \neq 0}} \frac{L(s+\mathrm{i} 2 \pi k)}{g_{k}^{\left(s_{0}\right)} \mathrm{e}^{s \theta_{k}^{\left(s_{0}\right)}}}=\left(\prod_{0<|k|<k_{\mathcal{T}}^{ \pm}} \frac{L(s+\mathrm{i} 2 \pi k)}{g_{k}^{\left(s_{0}\right)} \mathrm{e}^{s \theta_{k}^{\left(s_{0}\right)}}}\right)\left(\prod_{|k| \geq k_{\mathcal{T}}^{ \pm}} \mathrm{e}^{q^{\left(s_{0}\right)}(s, k)}\right)$.

The term $\prod_{0<|k|<k_{\mathcal{T}}^{ \pm}} \frac{L(s+\mathrm{i} 2 \pi k)}{g_{k}^{\left(s_{0}\right)} \exp \left(s \theta_{k}^{\left(s_{0}\right)}\right)}$ consists of a finite number of bounded terms (Condition (ii) of Definition 2.1) and is therefore bounded for all $s \in \mathcal{T}$. The second term can also be bounded by using Lemma 4.3 as

$$
\begin{aligned}
\left|q^{\left(s_{0}\right)}(s, k)\right| & \leq \frac{c\left|s-s_{0}\right|^{2}}{\min \left(\left|\operatorname{Im}\left(s_{0}\right)+2 \pi k\right|^{1+\epsilon},|\operatorname{Im}(s)+2 \pi k|^{1+\epsilon}\right)} \\
& \leq \frac{c d_{\mathcal{T}}^{\left(s_{0}\right)}}{\left(2 \pi\left(|k|-k_{\mathcal{T}}^{ \pm}+1\right)\right)^{1+\epsilon}} .
\end{aligned}
$$

Since $\sum_{|k| \geq k_{\mathcal{T}}^{ \pm}}\left(|k|-k_{\mathcal{T}}^{ \pm}+1\right)^{-(1+\epsilon)}$ is convergent, we invoke Weierstrass' M-test to conclude that $\sum_{|k| \geq k_{\mathcal{T}}^{ \pm}} q^{\left(s_{0}\right)}(s, k)$ is uniformly convergent on 
$s \in \mathcal{T}$. This, in turn, demonstrates the uniform convergence of the infinite product in (33) on $s \in \mathcal{T}$. Moreover, since $\sum_{|k| \geq k_{\mathcal{T}}^{ \pm}} q^{\left(s_{0}\right)}(s, k)$ converges to a finite value, $\prod_{|k| \geq k_{\mathcal{T}}^{ \pm}} \exp \left(q^{\left(s_{0}\right)}(s, k)\right)$ is non-zero. Consequently, the converging value of the infinite product becomes zero only if $L(s+\mathrm{i} 2 \pi k)=0$ for some $|k|<k_{\mathcal{T}}^{ \pm}$.

\section{Periodicity}

Next, we investigate how to set $\theta_{0}^{\left(s_{0}\right)}$ to obtain an i2 $\pi$-periodic $L_{\mathrm{d}}(s)$ function. Let us denote the truncated products by

$$
L_{\mathrm{d}, n}(s)=\mathrm{e}^{\frac{\mathrm{i} \sigma}{2 \pi} s^{2}} \prod_{k=-n}^{n} \frac{L(s+\mathrm{i} 2 \pi k)}{g_{k}^{\left(s_{0}\right)} \mathrm{e}^{s \theta_{k}^{\left(s_{0}\right)}}} .
$$

This shows that

$$
\begin{aligned}
& \frac{L_{\mathrm{d}, n}(s+\mathrm{i} 2 \pi)}{\mathrm{e}^{\frac{\mathrm{i} \sigma}{2 \pi}(s+\mathrm{i} 2 \pi)^{2}}}=\prod_{k=-n}^{n} \frac{L(s+\mathrm{i} 2 \pi(k+1))}{g_{k}^{\left(s_{0}\right)} \mathrm{e}^{(s+\mathrm{i} 2 \pi) \theta_{k}^{\left(s_{0}\right)}}} \\
& =\prod_{k=-n}^{n}\left(\frac{L(s+\mathrm{i} 2 \pi(k+1))}{g_{k+1}^{\left(s_{0}\right)} \mathrm{e}^{s_{k+1}^{\left(s_{0}\right)}}} \frac{g_{k+1}^{\left(s_{0}\right)}}{g_{k}^{\left(s_{0}\right)} \mathrm{e}^{\mathrm{i} 2 \pi \theta_{k}^{\left(s_{0}\right)}}} \mathrm{e}^{s\left(\theta_{k+1}^{\left(s_{0}\right)}-\theta_{k}^{\left(s_{0}\right)}\right)}\right) \\
& =\prod_{k=-n+1}^{n+1} \frac{L(s+\mathrm{i} 2 \pi k)}{g_{k}^{\left(s_{0}\right)} \mathrm{e}^{s \theta_{k}^{\left(s_{0}\right)}}} \prod_{k=-n}^{n} \frac{g_{k+1}^{\left(s_{0}\right)}}{g_{k}^{\left(s_{0}\right)} \mathrm{e}^{\mathrm{i} 2 \pi \theta_{k}^{\left(s_{0}\right)}}} \mathrm{e}^{s\left(\theta_{n+1}^{\left(s_{0}\right)}-\theta_{-n}^{\left(s_{0}\right)}\right)} \text {. }
\end{aligned}
$$

Since we are interested in the asymptotic behavior of $L_{\mathrm{d}, n}$ as $n \rightarrow+\infty$, we assume $n \geq \max \left(k_{0},\left|k^{+}\left(s_{0}, s\right)\right|,\left|k^{-}\left(s_{0}, s\right)\right|\right)$. This enables us to write

$$
\begin{aligned}
L_{\mathrm{d}, n}(s+\mathrm{i} 2 \pi)= & L_{\mathrm{d}, n}(s) \mathrm{e}^{-\mathrm{i} 2 \pi \sigma} \mathrm{e}^{s\left(\theta_{n+1}^{\left(s_{0}\right)}-\theta_{-n}^{\left(s_{0}\right)}-2 \sigma\right)} \\
& \times \underbrace{\frac{L(s+\mathrm{i} 2(n+1) \pi)}{g_{n+1}^{\left(s_{0}\right)} \mathrm{e}^{s \theta_{n+1}^{\left(s_{0}\right)}}}}_{\exp \left(q^{\left(s_{0}\right)}(s, n+1)\right)} \underbrace{\frac{g_{-n}^{\left(s_{0}\right)} \mathrm{e}^{s \theta_{-n}^{\left(s_{0}\right)}}}{L(s-\mathrm{i} 2 \pi n)}}_{\exp \left(-q^{\left(s_{0}\right)}(s,-n)\right)} \underbrace{\prod_{|k|<k_{0}} \frac{g_{k+1}^{\left(s_{0}\right)}}{g_{k}^{\left(s_{0}\right)} \mathrm{e}^{\mathrm{i} 2 \pi \theta_{k}^{\left(s_{0}\right)}}}}_{g_{k_{0}} \exp \left(-\mathrm{i} 2 \pi \theta_{0}^{\left(s_{0}\right)}\right)} \\
& \times \underbrace{\exp \left(\sum_{\substack{k=-n \\
|k| \geq k_{0}}}^{n+1} q^{\left(s_{0}\right)}\left(s_{0}+\mathrm{i} 2 \pi, k\right)\right)}_{A[n]} .
\end{aligned}
$$


According to Lemma 4.3, both $\exp \left(q^{\left(s_{0}\right)}(s, n+1)\right)$ and $\exp \left(-q^{\left(s_{0}\right)}(s,-n)\right)$ converge to 1 as $n \rightarrow+\infty$. Similarly, $A[n]$ converges to a finite and non-zero value, namely, $A_{\infty}$. Finally, Lemma 4.2 implies that $\theta_{n}^{\left(s_{0}\right)}$ and $\theta_{-n}^{\left(s_{0}\right)}$ converge as $n \rightarrow \infty$, and the difference of their limits is equal to $2 \sigma$. This results in $\lim _{n \rightarrow \infty} \exp \left(\left(\theta_{n+1}^{\left(s_{0}\right)}-\theta_{-n}^{\left(s_{0}\right)}-2 \sigma\right) s\right)=1$. This leads to the conclusion that, if $L_{\mathrm{d}}(s) \neq 0$, then

$$
\frac{L_{\mathrm{d}}(s+\mathrm{i} 2 \pi)}{L_{\mathrm{d}}(s)}=\lim _{n \rightarrow+\infty} \frac{L_{\mathrm{d}, n}(s+\mathrm{i} 2 \pi)}{L_{\mathrm{d}, n}(s)}=g_{k_{0}} \exp \left(-\mathrm{i} 2 \pi\left(\theta_{0}^{\left(s_{0}\right)}+\sigma\right)\right) A_{\infty},
$$

which is nonzero and does not depend on $s$. Thus, $\theta_{0}^{\left(s_{0}\right)} \in \mathbb{C}$ can be set such that $L_{\mathrm{d}}(s+\mathrm{i} 2 \pi)=L_{\mathrm{d}}(s)$. This proves $\mathrm{i} 2 \pi$-periodicity of $L_{\mathrm{d}}$.

\section{Uniqueness}

Assume that $\mathrm{L}_{\mathrm{d}}$ and $\widetilde{\mathrm{L}}_{\mathrm{d}}$ are two discretizations of $\mathrm{L}$ that satisfy Statements (i) and (ii) of Theorem 2.4 with constants $\left\{\sigma, g_{k}, \theta_{k}\right\}$ and $\left\{\tilde{\sigma}, \tilde{g}_{k}, \tilde{\theta}_{k}\right\}$, respectively. Also, let $\mathcal{B}_{\epsilon}\left(s^{*}\right) \subset R O C_{\mathrm{L}}$ be such that $L(s+\mathrm{i} 2 \pi k)$, and hence $L_{\mathrm{d}}(s)$ and $\widetilde{L}_{\mathrm{d}}(s)$ (due to Statement (ii)) never vanish for $s \in \mathcal{B}_{\epsilon}\left(s^{*}\right)$. Note that

$$
\frac{\widetilde{L}_{\mathrm{d}}(s)}{L_{\mathrm{d}}(s)}=\mathrm{e}^{\frac{\mathrm{i}(\tilde{\sigma}-\sigma)}{2 \pi} s^{2}} \prod_{k \in \mathbb{Z}} \alpha_{k} \mathrm{e}^{s \phi_{k}}
$$

and

$$
\frac{\widetilde{L}_{\mathrm{d}}\left(s_{1}\right) L_{\mathrm{d}}\left(s_{2}\right)}{L_{\mathrm{d}}\left(s_{1}\right) \widetilde{L}_{\mathrm{d}}\left(s_{2}\right)}=\mathrm{e}^{\frac{\mathrm{i}(\tilde{\sigma}-\sigma)}{2 \pi}\left(s_{1}^{2}-s_{2}^{2}\right)} \prod_{k \in \mathbb{Z}} \mathrm{e}^{\left(s_{1}-s_{2}\right) \phi_{k}},
$$

where $s, s_{1}, s_{2} \in \mathcal{B}_{\epsilon}\left(s^{*}\right)$ and $\alpha_{k}=\frac{g_{k}}{\tilde{g}_{k}}, \phi_{k}=\theta_{k}-\tilde{\theta}_{k}$. For large-enough $N \in \mathbb{N}$ so that $\frac{1}{N}<\epsilon$, it is possible to find $p_{1}, p_{2}, p_{3}, p_{4} \in \mathcal{B}_{\epsilon}\left(s^{*}\right)$ such that $p_{1}-p_{2}=\frac{1}{N}$ and $p_{3}-p_{4}=\frac{-\mathrm{i}}{N}$. By applying $(40)$ to $\left(s_{1}, s_{2}\right)=\left(p_{1}, p_{2}\right)$ and $\left(s_{1}, s_{2}\right)=\left(p_{3}, p_{4}\right)$, we obtain that

$$
\begin{gathered}
\prod_{k \in \mathbb{Z}} \mathrm{e}^{\frac{\phi_{k}}{N}}=\mathrm{e}^{\frac{\mathrm{i}(\tilde{\sigma}-\sigma)}{2 \pi}\left(p_{2}^{2}-p_{1}^{2}\right)} \frac{\widetilde{L}_{\mathrm{d}}\left(p_{1}\right) L_{\mathrm{d}}\left(p_{2}\right)}{L_{\mathrm{d}}\left(p_{1}\right) \widetilde{L}_{\mathrm{d}}\left(p_{2}\right)} \neq 0, \\
\prod_{k \in \mathbb{Z}} \mathrm{e}^{-\mathrm{i} \frac{\phi_{k}}{N}}=\mathrm{e}^{\frac{\mathrm{i}(\tilde{\sigma}-\sigma)}{2 \pi}\left(p_{4}^{2}-p_{3}^{2}\right)} \frac{\widetilde{L}_{\mathrm{d}}\left(p_{3}\right) L_{\mathrm{d}}\left(p_{4}\right)}{L_{\mathrm{d}}\left(p_{3}\right) \widetilde{L}_{\mathrm{d}}\left(p_{4}\right)} \neq 0 .
\end{gathered}
$$


By raising the modulus of the equations in (41) to the $N$ th power, we observe that both $\prod_{k \in \mathbb{Z}} \exp \left(\operatorname{Re}\left(\phi_{k}\right)\right)$ and $\prod_{k \in \mathbb{Z}} \exp \left(\operatorname{Im}\left(\phi_{k}\right)\right)$ converge to positive real numbers. This implies that both $\sum_{k \in \mathbb{Z}} \operatorname{Re}\left(\phi_{k}\right)$ and $\sum_{k \in \mathbb{Z}} \operatorname{Im}\left(\phi_{k}\right)$ converge to finite values; thus, $\sum_{k \in \mathbb{Z}} \phi_{k}=\phi$, where $\phi \in \mathbb{C}$ is finite. This further suggests that

$$
\prod_{k \in \mathbb{Z}} \mathrm{e}^{s \phi_{k}}=\mathrm{e}^{s \phi}
$$

Due to (39), $\prod_{k \in \mathbb{Z}} \alpha_{k}$ should also converge to a finite $\alpha \in \mathbb{C}$. In summary, we have that

$$
\frac{\widetilde{L}_{\mathrm{d}}(s)}{L_{\mathrm{d}}(s)}=\alpha \mathrm{e}^{\frac{\mathrm{i}(\tilde{\sigma}-\sigma)}{2 \pi} s^{2}} \mathrm{e}^{s \phi} .
$$

Since both $L_{\mathrm{d}}$ and $\widetilde{L}_{\mathrm{d}}$ are i $2 \pi$-periodic, their ratio should also be so, and it follows that $\tilde{\sigma}=\sigma$ and $\phi \in \mathbb{Z}$.

\subsection{Proof of Theorem 2.7}

Proof. In Theorem 2.4, by assuming that the transfer function $L(s)$ is holomorphic in most of its ROC, we showed that it can be discretized using the infinite product (2). The result then carries over directly to a frequency response $\widehat{L}(\omega)$ by setting $s=\mathrm{i} \omega$. However, one can check that only secondorder derivatives of $\widehat{L}(\omega)$ are referred to in the proof of Theorem 2.4. Thus, for a real-variable function $\widehat{L}(\omega)$, second-order differentiability (in contrast to holomorphicity) is sufficient to define a discretized operator. Furthermore, since in Definition 2.3 we assume that $\widehat{L}$ is continuous everywhere, Condition (ii) of Definition 2.1 is automatically fulfilled. Therefore, Theorem 2.4 can be invoked here to justify (3).

Since $\widehat{L}(\omega)$ is continuous, the uniform convergence of the infinite product proved in Theorem 2.4 establishes the continuity of $\widehat{L}_{\mathrm{d}}(\omega)$ and, similarly, the continuity of

$$
\widehat{\beta_{\mathrm{L}}}(\omega)=\frac{\widehat{L}_{\mathrm{d}}(\omega)}{\widehat{L}(\omega)}=\frac{\mathrm{e}^{-\frac{\mathrm{i} \sigma}{2 \pi} \omega^{2}}}{g_{0} \mathrm{e}^{\mathrm{i} \omega \theta_{0}}} \prod_{\substack{k \in \mathbb{Z} \\ k \neq 0}} \frac{\widehat{L}(w+2 \pi k)}{g_{k} \mathrm{e}^{\mathrm{i} \omega \theta_{k}}}
$$


It is known that the Riesz-basis property of $\beta_{\mathrm{L}}$ in (5) is equivalent to

$$
m \leq \sum_{n \in \mathbb{Z}}\left|\widehat{\beta_{\mathrm{L}}}(\omega+2 \pi n)\right|^{2} \leq M .
$$

To prove the left and right inequalities, we first bound $\widehat{\beta_{\mathrm{L}}}(\omega)$. For this purpose, let

$$
\begin{gathered}
M_{\widehat{\beta_{\mathrm{L}}}, \Omega}=\max \left\{\left|\widehat{\beta_{\mathrm{L}}}(\omega)\right| \mid-\Omega \leq \omega \leq \Omega\right\}, \\
M_{\widehat{L}_{\mathrm{d}}}=\max \left\{\left|\widehat{L}_{\mathrm{d}}(\omega)\right| \mid 0 \leq \omega \leq 2 \pi\right\},
\end{gathered}
$$

where $\Omega$ is introduced in Definition 2.3. Because $\widehat{\beta_{\mathrm{L}}}$ and $\widehat{L}_{\mathrm{d}}$ are continuous, the above maxima are well-defined and finite. Moreover, $M_{\widehat{L}_{\mathrm{d}}}$ is a uniform bound on $\widehat{L}_{\mathrm{d}}(\omega)$ since $\widehat{L}_{\mathrm{d}}$ is $2 \pi$-periodic. This suggests that

$$
\forall \omega,|\omega|>\Omega: \quad\left|\widehat{\beta_{\mathrm{L}}}(\omega)\right|=\frac{\left|\widehat{L}_{\mathrm{d}}(\omega)\right|}{|\widehat{L}(\omega)|} \leq \frac{M_{\widehat{L}_{\mathrm{d}}}}{c_{2}|\omega|^{0.5+\epsilon_{2}}} .
$$

This bound enables us to write

$$
\begin{aligned}
P\left\{\widehat{\beta_{\mathrm{L}}}\right\}(\omega) & :=\sum_{n \in \mathbb{Z}}\left|\widehat{\beta_{\mathrm{L}}}(\omega+2 \pi n)\right|^{2} \\
& \leq \sum_{\frac{-\Omega-\omega}{2 \pi} \leq n \leq \frac{\Omega-\omega}{2 \pi}} M_{\widehat{\beta_{\mathrm{L}}}, \Omega}+\frac{M_{\widehat{L_{\mathrm{d}}}}^{2}}{c_{2}^{2}} \sum_{\substack{n<\frac{-\Omega-\omega}{2 \pi} \\
n>\frac{\Omega-\omega}{2 \pi}}} \frac{1}{|\omega+2 \pi n|^{1+2 \epsilon_{2}}} \\
& \leq \frac{\Omega+\pi}{\pi} M_{\widehat{\beta_{\mathrm{L}}}, \Omega}+\frac{2 M_{\widehat{L_{\mathrm{d}}}}^{2}}{c_{2}^{2}} \sum_{n=0}^{\infty} \frac{1}{(\Omega+2 \pi|n|)^{1+2 \epsilon_{2}}}<\infty .
\end{aligned}
$$

As the upperbound does not depend on $\omega$, the series $\sum_{n \in \mathbb{Z}}\left|\widehat{\beta_{\mathrm{L}}}(\omega+2 \pi n)\right|^{2}$ is uniformly convergent. Hence, due to the continuity of $\left|\widehat{\beta_{\mathrm{L}}}(\omega+2 \pi n)\right|^{2}$, $P\left\{\widehat{\beta_{\mathrm{L}}}\right\}(\omega)$ is also continuous. The fact that $P\left\{\widehat{\beta_{\mathrm{L}}}\right\}(\omega)$ is periodic confirms that it attains its maximum and minimum values and that these values are finite (continuity).

According to the definition, we have that $P\left\{\widehat{\beta_{\mathrm{L}}}\right\}(\omega) \geq 0$. Thus, to complete the proof we only need to show that $P\left\{\widehat{\beta_{\mathrm{L}}}\right\}$ never vanishes $(i . e$., that the minimum value is strictly positive). Assume $P\left\{\widehat{\beta_{\mathrm{L}}}\right\}\left(\omega_{0}\right)=0$ for 
some $\omega_{0} \in[0,2 \pi)$. As a result, we should have $\widehat{L}_{\mathrm{d}}\left(\omega_{0}+2 \pi n\right)=0$ for all $n \in \mathbb{Z}$. On one hand, Theorem 2.4 states that $\widehat{L}_{\mathrm{d}}\left(\omega_{0}+2 \pi n\right)$ vanishes only if $\widehat{L}\left(\omega_{0}+2 \pi n+2 \pi k\right)$ vanishes for at least one value of $k \neq 0$. On the other hand, Condition (i) of Definition 2.3 restricts the number of zeros among $\widehat{L}\left(\omega_{0}+2 \pi n+2 \pi k\right)$ to be at most one. Hence, there is a unique $k^{*} \neq 0$ such that $\widehat{L}\left(\omega_{0}+2 \pi n+2 \pi k^{*}\right)=0$. This, however, implies that $\widehat{L}_{\mathrm{d}}\left(\omega_{0}+2 \pi\left(n+k^{*}\right)\right) \neq 0$, since $\widehat{L}\left(\omega_{0}+2 \pi\left(n+k^{*}\right)+2 \pi k\right) \neq 0$ for all $k \neq 0$. This contradicts the assumption that $P\left\{\widehat{\beta_{\mathrm{L}}}\right\}\left(\omega_{0}\right)=0$.

\section{Acknowledgement}

This work was support by Swiss National Science Foundation under the grant 200020-162343.

\section{Appendix A. Proof of Property 3.3}

Proof. The facts that $\widehat{L}(\omega)$ is continuous and $\frac{\widehat{L}(\omega)}{|\widehat{L}(\omega)|} \neq u$ show that we can define the phase for $\widehat{L}(\omega)$ continuously, by modifying the range as $\arg ^{*}(c) \in$ $\left[\measuredangle u, \measuredangle u+2 \pi\left[\right.\right.$. Thus, the frequency multiplier $\widehat{L}_{z_{0}}(\omega)$ defined in Property 3.3 is continuous. Similarly, we can show that, at those values of $\omega$ for which $\widehat{L}(\omega)$ is nonzero and differentiable, $\widehat{L}_{z_{0}}(\omega)$ is also nonzero and differentiable. Furthermore,

$$
\frac{\mathrm{d}}{\mathrm{d} \omega} \log \widehat{L}_{z_{0}}(\omega)=z_{0} \frac{\mathrm{d}}{\mathrm{d} \omega} \log \widehat{L}(\omega)
$$

Thus, $\widehat{L}_{z_{0}}$ satisfies Conditions (i) and (ii) of Definition 2.3. In addition, note that

$$
\begin{aligned}
\left|\widehat{L}_{z_{0}}(\omega)\right| & =\exp \left(\operatorname{Re}\left(z_{0}\right) \log |\widehat{L}(\omega)|-\operatorname{Im}\left(z_{0}\right) \arg (\widehat{L}(\omega))\right) \\
& \geq|\widehat{L}(\omega)|^{\operatorname{Re}\left(z_{0}\right)} \mathrm{e}^{-\pi \operatorname{Im}\left(z_{0}\right)} \geq\left(c_{2} \mathrm{e}^{-\pi \operatorname{Im}\left(z_{0}\right)}\right)|\omega|^{\frac{1}{2}+\epsilon_{2}} .
\end{aligned}
$$

Hence, $\widehat{L}_{z_{0}}$ also fulfills Condition (iii) and leads to the construction of the $z_{0}$ th-order B-spline $\beta_{\mathrm{L}_{z_{0}}}$. 


\section{Appendix B. Evaluation of the Infinite Product in Example 3.5}

By setting the parameters as in (12), for $0 \neq k \in \mathbb{Z}$ we shall have

$$
\begin{aligned}
\frac{\widehat{L}_{z_{0}}(\omega+2 \pi k)}{g_{k} \mathrm{e}^{\mathrm{i} \omega \theta_{k}}} & =\frac{(\mathrm{i}(\omega+2 \pi k)-\lambda)^{z_{0}}}{(\mathrm{i} 2 \pi k)^{z_{0}}} \mathrm{e}^{\mathrm{i} \frac{\mathrm{i} \omega-\lambda}{2 \pi k} z_{0}} \\
& =\left|1-\mathrm{i} \frac{\mathrm{i} \omega-\lambda}{2 \pi k}\right|^{z_{0}} \exp \left(\mathrm{i} z_{0}\left(\frac{\pi}{2} \operatorname{sign}(k)-\tan ^{-1}\left(\frac{\omega+2 \pi k-\operatorname{Im}(\lambda)}{\operatorname{Re}(\lambda)}\right)\right)\right) \mathrm{e}^{\mathrm{i} \frac{\mathrm{i} \omega-\lambda}{2 \pi k} z_{0}} .
\end{aligned}
$$

By taking $|k|$ large enough for $k$ and $\omega+2 \pi k-\operatorname{Im}(\lambda)$ to have the same sign, we ensure that

$$
\left.-\frac{\pi}{2}<\frac{\pi}{2} \operatorname{sign}(k)-\tan ^{-1}\left(\frac{\omega+2 \pi k-\operatorname{Im}(\lambda)}{\operatorname{Re}(\lambda)}\right)\right)<\frac{\pi}{2} .
$$

For these values of $k$, we have that

$$
\frac{\widehat{L}_{z_{0}}(\omega+2 \pi k)}{g_{k} \mathrm{e}^{\mathrm{i} \omega \theta_{k}}}=(1-\underbrace{\mathrm{i} \frac{\mathrm{i} \omega-\lambda}{2 \pi k}}_{\eta_{k}})^{z_{0}} \mathrm{e}^{\mathrm{i} \frac{\mathrm{i} \omega-\lambda}{2 \pi k} z_{0}}=\left(1-\eta_{k}\right)^{z_{0}} \mathrm{e}^{\eta_{k} z_{0}},
$$

where we interpret $\left(1-\eta_{k}\right)^{z_{0}}$ using the standard definition of $\arg (c) \in[-\pi, \pi[$. Now, using the binomial expansion of $\left(1-\eta_{k}\right)^{z_{0}}$ and the Taylor series of $\mathrm{e}^{\eta_{k} z_{0}}$, we can again verify that

$$
\frac{\widehat{L}_{z_{0}}(\omega+2 \pi k)}{g_{k} \mathrm{e}^{\mathrm{i} \omega \theta_{k}}}-1=\mathcal{O}\left(\frac{1}{k^{2}}\right)
$$

which justifies the convergence of the infinite product. In order to evaluate the limit, we note that

$$
\begin{aligned}
& \frac{\widehat{L}_{z_{0}}(\omega+2 \pi k) \widehat{L}_{z_{0}}(\omega-2 \pi k)}{g_{k} \mathrm{e}^{\mathrm{i} \omega \theta_{k}} g_{-k} \mathrm{e}^{\mathrm{i} \omega \theta_{-k}}}=\frac{(\mathrm{i}(\omega+2 \pi k)-\lambda)^{z_{0}}(\mathrm{i}(\omega-2 \pi k)-\lambda)^{z_{0}}}{(\mathrm{i} 2 \pi k)^{z_{0}}(-\mathrm{i} 2 \pi k)^{z_{0}}} \\
& =\left|1-\frac{\left(\frac{\mathrm{i} \omega-\lambda}{2 \mathrm{i}}\right)^{2}}{(k \pi)^{2}}\right|^{z_{0}} \exp \left(-\mathrm{i} z_{0}\left(\tan ^{-1}\left(\frac{\omega+2 \pi k-\operatorname{Im}(\lambda)}{\operatorname{Re}(\lambda)}\right)+\tan ^{-1}\left(\frac{\omega-2 \pi k-\operatorname{Im}(\lambda)}{\operatorname{Re}(\lambda)}\right)\right)\right) .
\end{aligned}
$$

Thus,

$$
\begin{aligned}
\widehat{L}_{z_{0}, \mathrm{~d}}(\omega)= & \prod_{k \in \mathbb{Z}} \frac{\widehat{L}_{z_{0}}(\omega+2 \pi k)}{g_{k} \mathrm{e} \mathrm{e}^{\mathrm{i} \omega \theta_{k}}} \\
= & \mathrm{e}^{-\frac{\lambda z_{0}}{2}}\left|\mathrm{e}^{\frac{\mathrm{i} \omega-\lambda}{2}}-\mathrm{e}^{-\frac{\mathrm{i} \omega-\lambda}{2}}\right|^{z_{0}} \exp \left(\mathrm { i } z _ { 0 } \left(\frac{\omega}{2}-\tan ^{-1}\left(\frac{\omega-\operatorname{Im}(\lambda)}{\operatorname{Re}(\lambda)}\right)-\right.\right. \\
& \left.\left.\sum_{k=1}^{\infty}\left[\tan ^{-1}\left(\frac{\omega+2 \pi k-\operatorname{Im}(\lambda)}{\operatorname{Re}(\lambda)}\right)+\tan ^{-1}\left(\frac{\omega-2 \pi k-\operatorname{Im}(\lambda)}{\operatorname{Re}(\lambda)}\right)\right]\right)\right) .
\end{aligned}
$$


The infinite sum of the inverse tangents in the above equation is convergent as

$$
\begin{aligned}
\tan ^{-1}\left(\frac{\omega+2 \pi k-\operatorname{Im}(\lambda)}{\operatorname{Re}(\lambda)}\right)+\tan ^{-1}\left(\frac{\omega-2 \pi k-\operatorname{Im}(\lambda)}{\operatorname{Re}(\lambda)}\right) & =\tan ^{-1}\left(\frac{2 \operatorname{Re}(\lambda)(\omega-\operatorname{Im}(\lambda))}{(\operatorname{Re}(\lambda))^{2}-(\omega-\operatorname{Im}(\lambda))^{2}+(2 \pi k)^{2}}\right) \\
& =\mathcal{O}\left(\frac{1}{k^{2}}\right),
\end{aligned}
$$

for all $k \geq \frac{|\omega-\operatorname{Im}(\lambda)|}{2 \pi}$. Moreover, we can verify that

$$
\tan ^{-1}\left(\frac{x}{\alpha}\right)+\sum_{k=1}^{\infty}\left(\tan ^{-1}\left(\frac{x+2 \pi k}{\alpha}\right)+\tan ^{-1}\left(\frac{x-2 \pi k}{\alpha}\right)\right)=\frac{1}{2} \int_{0}^{x} \frac{\sinh (\alpha)}{\cosh (\alpha)-\cos (\tau)} \mathrm{d} \tau
$$

by differentiating both sides with respect to $x$ (also note that both sides vanish at $x=0$ ). It is trivial to check that the integral on the right-hand side increases by $\pi$ whenever $x$ increases by $2 \pi$. Hence,

$$
\phi(x) \triangleq \frac{x}{2}-\frac{1}{2} \int_{0}^{x} \frac{\sinh (\alpha)}{\cosh (\alpha)-\cos (\tau)} \mathrm{d} \tau
$$

is a $2 \pi$-periodic function. The closed form of $\phi(x)$ for $|x| \leq \pi$ is also given by

$$
|x| \leq \pi: \quad \phi(x)=\frac{x}{2}-\tan ^{-1}\left(\operatorname{coth}\left(\frac{\alpha}{2}\right) \tan \left(\frac{x}{2}\right)\right)=\arg ^{*}\left(\mathrm{e}^{\frac{\alpha}{2}}-\mathrm{e}^{\mathrm{i} x-\frac{\alpha}{2}}\right)-\pi .
$$

Now, by plugging this result into (B.1), the simplified form of (13) can be achieved by basic algebraic manipulations.

Remark Appendix B.1. In the previous example of $\widehat{L}_{z_{0}}(\omega)=(\mathrm{i} \omega-\lambda)^{z_{0}}$ with $\operatorname{Re}\left(z_{0}\right) \geq 1$ and $\operatorname{Re}(\lambda) \geq 0$, if we interpret $c^{z_{0}}$ using the standard definition of $\arg (c) \in[-\pi, \pi[$, we obtain that

$$
\widehat{L}_{z_{0}}(\omega)= \begin{cases}|\mathrm{i} \omega-\lambda|^{z_{0}} \exp \left(\mathrm{i} z_{0}\left(\pi-\tan ^{-1}\left(\frac{\omega-\operatorname{Im}(\lambda)}{\operatorname{Re}(\lambda)}\right)\right)\right), & \omega>\operatorname{Im}(\lambda), \\ |\mathrm{i} \omega-\lambda|^{z_{0}} \exp \left(-\mathrm{i} z_{0}\left(\pi+\tan ^{-1}\left(\frac{\omega-\operatorname{Im}(\lambda)}{\operatorname{Re}(\lambda)}\right)\right),\right. & \omega \leq \operatorname{Im}(\lambda),\end{cases}
$$

which contains a single discontinuity at $\omega=\operatorname{Im}(\lambda)$. One can check that this operator is still discretizable according to Definition 2.1. Also, with parameters similar to (12) except for $g_{0}=\mathrm{e}^{-\lambda z_{0} / 2}$ and $\theta_{0}=\frac{z_{0}}{2}$, we can demonstrate that

$$
\widehat{L}_{z_{0}, \mathrm{~d}}(\omega)=\left(1-\mathrm{e}^{\mathrm{i} \omega-\lambda}\right)^{z_{0}} \exp \left(\mathrm{i} 2 \pi z_{0}\left(\frac{1}{2}-\left\{\frac{\omega-\operatorname{Im}(\lambda)}{2 \pi}\right\}\right)\right)
$$

where $\{x\}$ stands here for the fractional part of $x$. 


\section{References}

[1] Christensen, O., Massopust, O., 2012. Exponential B-splines and the partition of unity property. Adv. Comput. Math. 37 (3), 301-318.

[2] Dahmen, W., Micchelli, C., 1987. On theory and application of exponential splines. In: Chui, C., Shumaker, L., Utreras, F. (Eds.), Topics in Multivariate Approximation. Academic Press, New York, pp. 37-46.

[3] de Boor, C., 1972. On calculating with B-splines. J. Approx. Theory $6(1), 50-62$.

[4] de Boor, C., 1978. A practical guide to splines. Springer, New York.

[5] Forster, B., Unser, M., Blu, T., 2006. Complex B-splines. Appl. Comp. Harmon. Anal. 20, 261-282.

[6] Massopust, P., 2014. Exponential splines of complex order. Contemporary Mathematics 626, 87-106.

[7] Micchelli, C., 1976. Cardinal L-spline. In: Karlin, S., Micchelli, C., Pinkus, A., Schoenberg, I. J. (Eds.), Studies in Spline Functions and Approximation Theory. Academic Press, pp. 203-250.

[8] Ron, A., 1988. Exponential box splines. Constructive Approximation 4 (1), 357-378.

[9] Sarason, D., 2007. Complex function theory, 2nd Edition. American Mathematical Society.

[10] Schoenberg, I. J., 1946. Contribution to the problem of approximation of equidistant data by analytic functions, Part A: On the problem of smoothing or graduation, a first class of analytic approximation formulas. Quart. Appl. Math. 4 (1), 45-99.

[11] Schumaker, L. L., 2007. Spline functions: Basic theory, 3rd Edition. Cambridge University Press.

[12] Unser, M., Blu, T., 2000. Fractional splines and wavelets. SIAM Rev. $42(1), 43-67$.

[13] Unser, M., Blu, T., Apr. 2005. Cardinal exponential splines: Part ITheory and filtering algorithms. IEEE Trans. Sig. Proc. 53 (4), 14251449. 